\title{
Parkinson's Disease: Clinical Signs and Symptoms, Neural Mechanisms, Positron Emission Tomography, and Therapeutic Interventions
}

\author{
K.L. Leenders ${ }^{1}$ and W.H. Oertel ${ }^{2}$ \\ ${ }^{1}$ Groningen University Hospital, Department of Neurology, Hanzeplein 1 \\ PO Box 30.001, 9700 RB Groningen, The Netherlands; \\ ${ }^{2}$ Philipps-University Marburg, Klinikum für Neurologie, 35039 Marburg, Germany
}

\section{SUMMARY}

Parkinson's disease is one of the most frequent neurodegenerative brain diseases. Its time course is slow and is characterized by progressive loss of dopaminergic and other brainstem neurons resulting in malfunctioning of the cerebral neuronal systems responsible for motor functions. The clinical signs are slowness of movement, muscle rigidity and rest-tremor amongst other features. The cause of the disease is unknown, but recently involvement of genetic factors is being researched. Positron emission tomography (PET) allows in vivo determination of striatal dopaminergic activity. This has increased our insight in the pathophysiology of the disease and permits direct study of disease progression at a biochemical level and equally to monitor whether potential neuroprotective interventions are indeed effective. Thus far no drug has emerged but promising substances are currently being studied.

\section{KEYWORDS}

Parkinson's disease, FDOPA-PET, gene mutation, disease progression, neuroprotection

\section{INTRODUCTION}

Parkinson's disease (PD) has for the first time been described in a comprehensive way in 1817 by James Parkinson in an article entitled "An Essay on the Shaking Palsy". The disorder has since then been named after him. The diagnosis PD is made on clinical grounds solely since there does not exist a specific laboratory test to confirm the characteristic cerebral pathological alterations which lie at the basis of this condition. In most cases the clinical pattern of signs and symptoms is sufficiently clear to make the diagnosis with confidence. However, a certain number of patients will pose considerable difficulties as to the correct diagnosis, particularly in early stages of their disease, even for an experienced neurologist. Overlap with diseases which are not PD, but are accompanied with some form of parkinsonistic features, may occur regularly.

In PD at least two of the three cardinal signsbradykinesia, rigidity and tremor-need to be present (Levy \& Cummings, 1999). The tremor is typically prominent when the patient is at rest. In addition, a large number of other signs and symptoms may be present at different stages of the disease and in individually varying composition or intensity: impaired postural reflexes, masked face, low speech volume, swallowing difficulty, micro 
graphia, flexed posture, small shuffling steps, movement initiation problems, freezing. The clinical picture often is further complicated by depression. Certain neuropsychological functional impairments and even a dementing condition may develop at later stages. The onset of PD is insidious and usually unilateral. Also with progression of the disease, when both body sides are affected, the asymmetries remain. Most commonly the disease starts at middle age with increasing prevalence above the age of 50 years, but 'young onset cases' are not uncommon. Progression is in most cases slow but inexorable. The endstage of the disease is reached after many years, usually more than ten.

In this chapter, apart from clinical features, some aspects of the pathophysiology of the disease will be described. In particular the role of positron emission tomography (PET) will be highlighted.

\section{CAUSE}

To date the cause of idiopathic Parkinson's disease is still unknown. Accelerated aging has been discussed as a possibility, but is now discarded mainly because the brainstem pathology found in post-mortem PD tissue is clearly different from the patterns of alteration seen with aging (Hirsch et al., 2000). Genetic and environmental factors have been investigated over the last decades without, as yet, a clear-cut unifying unimodal causative model. During the last few years, possible genetic influences have become the focus of attention. Only in a minority of cases PD occurs in a familial context, although an overall increased relative risk (compared to the population at large) of two to three for family members of PD patients to become afflicted has been found. This is comparable to the situation in Alzheimer's disease.

Mutation of the alpha-synuclein gene (chromosome $4 \mathrm{q} 21-23$ ) has been identified in one large
Italian family and in five Greek families. That mutation gave rise to an autosomal dominant pattern of inheritance of parkinsonism. The clinical expression of the disease, however, was not quite typical for PD in the case of the Ala53Thr point mutation of the chromosome. For instance, the disease manifested itself at early ages. The Ala30Pro point mutation, recently discovered in a German family, however, resulted in a syndrome which phenotypically was much more like that seen in idiopathic sporadic PD (Kruger et al., 2001).

Mutation of the Parkin gene (chromosome 6q25-27) has been identified as the cause of an autosomal-recessive juvenile form of parkinsonism, although the start of the disease at an early age and other atypical neuropsychological features again distinguish this condition somewhat from the 'common' form of PD. In the meantime, a spectrum of different alterations of the chromosome (various deletions or point mutations and others) have been found in the reported families. Thus, apparently a widely varying number of genetic pathological changes of several chromosomes can give rise to parkinsonism (Klein et al., 2000).

It needs to be realized, however, that in the overwhelming majority of familial cases of Parkinson's disease, or in sporadic PD patients for that matter, no genetic abnormality has been identified. In the majority of cases, therefore, it is believed that environmental factors, possibly on the basis of some form of genetic susceptibility, play a major hitherto unknown role. Some have suggested that the place of birth and residence seem to play a greater role than the race. AfroAmericans living in the U.S. have a nearly five times higher risk as Africans living in Nigeria. Afro-Americans and Caucasians living in the same community in the U.S. have similar risks of developing Parkinson's disease. 


\section{CLINICAL SIGNS AND SYMPTOMS}

The patients usually notice rest tremor or slowing of movements in one form or the other as a first sign or symptom. Rigidity in the form of stiffness and pain, on the other hand, is rarely reported as the main complaint by the patient, although it is a characteristic sign often to be found at patient examination. Rigidity is determined by passive movement by the examiner of the neck, upper and lower limbs of the patient. Increased muscle tone throughout the range of motion is looked for. If the rigidity is mild, it may be accentuated by letting the patient move the contralateral limb by opening and closing the hand. Rigidity should be distinguished from spasticity because the increase in tone in that condition is more prominent with the initiation of passive movement and is greater in one direction than in the other. Spasticity results from a lesion at the level of the upper motor neuron and is accompanied by an increased stretch reflex and other alterations. These latter findings are not seen in patients with rigidity.

Postural instability is not a presenting symptom or sign of PD but can certainly arise in the course of the disease. This needs to be tested specifically when the patient is examined in order to categorize the patient in the widely used Hoehn and Yahr scale: if the postural reflexes are affected, the patient is assigned to category III instead of II of a total five categories.

There are numerous other signs and symptoms of PD that are often brought to the physician's attention by the patient or family members. Micrographia is a common early sign, characterized by a slowness or smallness to the handwriting. Mask facies is another bradykinetic symptom characteristic of Parkinson's disease. Also, slowing of daily living activities occurs, including such activities as dressing, bathing, turning in bed, getting in and out of a chair. Well-known are the problems with gait in PD: some shuffling or dragging of a leg. The posture is often stooped with flexion of the knees, hips, trunk, and neck. Bradykinetic or hypophonic speech, which may require the patient to repeat himself frequently, is one of the bulbar symptoms. Drooling is also common and choking may occur. The loss of the sense of smell, or anosmia, in relation to alterations of the dopaminergic pathway projecting to the bulbus olfactorius, has been welldocumented in Parkinson's disease. It is also possible that patients will present with a foot dystonia, in which the foot will turn in and with involuntary curling of the toes, especially in the morning. This may be an unpleasant symptom, often accompanied by pain. If it occurs later in the disease it usually signifies a relative understimulation of the dopaminergic system due to low blood drug levels, particularly early in the morning when during the night no medication has been taken.

Idiopathic PD needs to be differentiated from other conditions causing parkinsonism (Levy \& Cummings, 1999). Certain drugs like neuroleptics but also others may induce parkinsonism. Atypical PD can occur in the context of other diseases like Progressive Supranuclear Palsy (PSP) or multiple system atrophy (MSA). If a supranuclear gaze palsy, particularly a downgaze palsy, is present, a PSP is more likely. In that condition, also frequent falls early in the disease are characteristic in addition to more axial (nuchal) rigidity compared to limb rigidity. The signs are often symmetrical, and a resting tremor is absent or only mild. Response to parkinsonian drugs is commonly poor or absent, although early on there may occasionally be a good effectivity. An MSA is suspected if involvement of the upper motor neuron or a cerebellar syndrome is found. Often the autonomic nervous system is affected too, resulting, for example, in micturition problems or orthostatic hypotension. Also MSA develops usually rather rapidly, symmetrically, and without much of a 
hypotension. Also MSA develops usually rather rapidly, symmetrically, and without much of a tremor and does not respond well on antiparkinsonian medication. Patients with early-onset or rapidly progressive dementia, accompanied with visual hallucinations not induced by medication, are more likely to have diffuse Lewy body disease or Alzheimer's disease. Patients with an early onset of urinary incontinence should be evaluated for normal pressure hydrocephalus.

Even though a patient with parkinsonism can usually be classified correctly during life, a definitive diagnosis of PD can be made only by autopsy (see below). Neuroimaging studies applying radiotracers during life can reliably demonstrate the presence of a presynaptic dopaminergic defect in the nigrostriatal neuronal system, but as such, this does not differentiate between the various forms of parkinsonism. Usage of a combination of various tracers, however, can help to clarify the biochemical changes and thus assist in making a diagnosis.

\section{PATHOLOGY}

A normal midbrain at post-mortem shows the substantia nigra (Latin for 'black substance') as dark black/brown colored areas between the cerebral peduncles and the nucleus ruber. This abundant pigmentation is caused by the neuromelanin accumulation in the cell bodies of the nigral dopaminergic neurons. In PD, the nigral dopaminergic neurons are gradually and to a certain extent lost, particularly in the lateral and ventral parts of the substantia nigra. Therefore, the midbrain of a PD patient looks bleak because of depigmentation. Microscopically there is also some gliosis in the substantia nigra in PD. Many lesioned dopaminergic neurons contain abnormal inclusion bodies, the Lewy body inclusions. These are characterized by a dense pink center and a lighter pink periphery. The combined loss of dopaminergic neurons in the substantia nigra, together with the inclusion of Lewy bodies, is seen as the pathological hall-mark of idiopathic PD.

There are speculations about why in PD usually only the lateral and ventral part of the substantia nigra is affected. It has been found that the glial cells in that particular part of the substantia nigra have different biochemical properties (Hirsch, 2000). Possibly in the case of PD, protective properties of glial cells in those regions of the substantia nigra are not operational or not sufficiently effective.

Various biochemical deficits or cell biological alterations in the brain tissue of PD patients have been found: mitochondrial complex $I$ is impaired in the substantia nigra, iron accumulation is increased, and signs of increased oxidative stress and reduced defenses against this stress have been found, also increased apoptosis has been reported. It is generally supposed that all these cellular phenomena are the expression of dysregulation relatively far down the cascade of events active in the impaired neurons in PD. The quest will be to find the key step(s) which initiates all the above mentioned pathological mechanisms.

\section{BASAL GANGLIA CIRCUITS}

The cerebral stations which plan and execute movements are organized in a complex way. Motor plans are stored, composed, and activated by many parietal and frontal cortical regions. In addition, two major systems interact with the cortical action to make movements possible. The cerebellum is necessary, for example, for obtaining accuracy and context-shaping of movements. The basal ganglia (mainly the striatum, pallidum, subthalamic nucleus, as well as the thalamus) have 
a role in learning automated skilled movements, but their role is much more difficult to formulate in physiological terms. They do seem to influence temporal and spatial discrimination.

Within the cortex, the neurons have dense and complicated cortico-cortical interconnections. In contrast, the connections between the cortex and striatum, between striatum and pallidum, between pallidum and thalamus, and between thalamus and cortex are all formed by single-layered elements. This has been the basis for the efforts to determine which synaptic transmission is excitatory or inhibitory via the type of neurotransmitter at the specific connections. Thus, a system of integrated influence, either activating or inhibiting, could be proposed for the basal ganglia system of corticosubcortico-cortical loops.

A central role in this system is played by the dopaminergic nigrostriatal projection, which modifies in various ways the transmission of the corticostriatal signals to the striato-pallidal projection neurons. Since the dopaminergic neuronal system is impaired in PD (see above), the basal ganglia circuits are therefore in disarray (Bergman et al., 1998). Ultimately this results in an altered firing pattern of the internal part of the globus pallidus to which the basal ganglia massively converge as major output station. In particular, the functional firing segregation between the pallidal neurons is lost, leading to oscillatory activity of the pallidum which is normally not present (Raz et al., 2001). The pallidum has, in turn, an enormous divergent connection with the cortex through the thalamus. It therefore is understandable that the pathologically altered firing pattern of the globus pallidus has a deeply disturbing influence on the cortical functions. The major projection of the basal ganglia is to the supplementary cortex, which is an important motor planning area: for example, initiation of movement patterns, organization of sequential movements, and other aspects are prepared there.

\section{CLINICAL PHYSIOLOGICAL CONSEQUENCES}

The clinical hallmarks of PD-akinesia (unable to start a movement), bradykinesia (slowness of movement), and tremor-can thus be traced to the arrangement of brain regions and their impaired function. PD patients show a slow reaction time and prolonged movement time. It appears that velocity modulation during movements is affected rather than an overall reduction of velocity. Sequential movements of PD patients are impaired, and also simultaneous actions are difficult to perform. An often observed phenomenon is that a patient has to stop a certain movement in order to be able to answer, when the patient is asked something during the performance of a movement. Apparent is the slowing of cognitive speed if choice complexity increases. On the other hand, the central processing of information is qualitatively preserved, for example, use of advance information for motor preparation.

\section{${ }^{18}$ F-FLUORODOPA PET}

Positron Emission Tomography (PET) is a radiotracer method using special computer tomographic equipment to register the signals emerging from the body after application of appropriate radiotracers. The radiolabel is an isotope of physiological atoms like carbon-11, oxygen-15 or fluor-18, and these have certain characteristics: they are short lived (minutes to hours) and emit two high-energy gamma rays in opposite directions, which enables regional quantitation of the signal at high sensitivity. A further feature of these tracers is that they usually are substances of a physiological nature like derivatives of glucose, amino acids, enzyme substrates, or receptor binding substances. Taken together, the method allows in a computer tomographic way to measure regional 
activity in vivo of biochemical or physiological processes in man or in experimental animals. This can be done for the whole body, but here we limit ourselves to the brain. Particularly for slowly progressing degenerative brain diseases like PD, such a method constitutes an important addition to the possibilities of investigating patients with this disease, since the brain is hardly accessible in a direct way.

Relevant for the study of PD is the possibility to use the tracer FDOPA (6-[F-18]-fluoro-L-dopa) and PET. This tracer serves as a substrate for the enzyme dopa-decarboxylase and is then accumulated as fluorodopamine in the dopaminergic nerve terminals. Particularly, the striatal capacity to decarboxylase and store this tracer can be performed quantitatively (Melega et al., 1991) In PD patients, this conveys the degree of dopaminergic nigrostriatal neuronal impairment since loss of nigral dopaminergic neurons will be accompanied by a loss of striatal dopaminergic nerve endings. However, also nigral dopaminergic neurons which still are present but do not function properly will most likely contribute to impaired endogenous nerve terminal function. The parameter to compare subjects can be a simple ratio between the activity in the target striatal regions and a nondopaminergic brain reference region, for example, occipital lobe or cerebellum.

Alternatively, a more complicated measure can be derived from the dynamic time curves of measured activity from the moment of application of the radiotracer onwards. From these tracerkinetic models a specific constant can be calculated reflecting specific regional tracer uptake. This constant is generally named $\mathrm{Ki}$. If the reference of tracer input is the arterial plasma then the unit should be written as $\mathrm{Ki}$ (plasma), but if the reference is the occipital lobe the unit may be denoted as Ki(occipital) or briefly Kocc. This unit is expressed as per minute $(1 / \mathrm{min})$.

In PD the decrease in specific FDOPA uptake is not uniformly distributed. The nigrostriatal neuronal system of the most affected part in the substantia nigra (lateral and ventral part) projects mainly to the putamen. In the cross-sectional images obtained via PET (see Fig. 1), it can be seen that indeed the posterior parts of the striatum show the largest decrease in FDOPA uptake while the caudate nucleus regions are relatively spared. In asymmetric PD patients the FDOPA uptake is usually also asymmetrically impaired: the contralateral side shows the highest decrease.

On average, in well established PD patients the specific FDOPA uptake in putamen is about $40 \%$ to $45 \%$ of that in healthy age-matched controls (Leenders et al., 1990).

\section{START OF DOPAMINERGIC DECLINE}

The decline of the striatal dopaminergic neuromodulation must have begun some time before the clinical signs and symptoms appear. There has been some speculation about how many years this period in general might be. Post-mortem investigations suggest about 4 years. The progression data obtained via FDOPA PET would support this contention (Leenders, 1997). From Fig. 2 it can be seen that indeed extrapolation from the estimated period of clinical duration of the disease in a group of patients towards the mean of healthy control values there is an approximate time lag of 5 to 6 years before the striatal dopaminergic capacity to accumulate FDOPA has decreased to a level when the signs and symptoms appear. It can also be seen that the striatal activity is not very low when the disease manifests itself clinically. One interpretation is, that the endogenous dopamine production is very low indeed, at a stage when there still are a sufficient number of nerve terminals left (although malfunctioning) to take up exogenous levodopa or FDOPA. It would explain why in the beginning of the disease levodopa medication is so effective. 


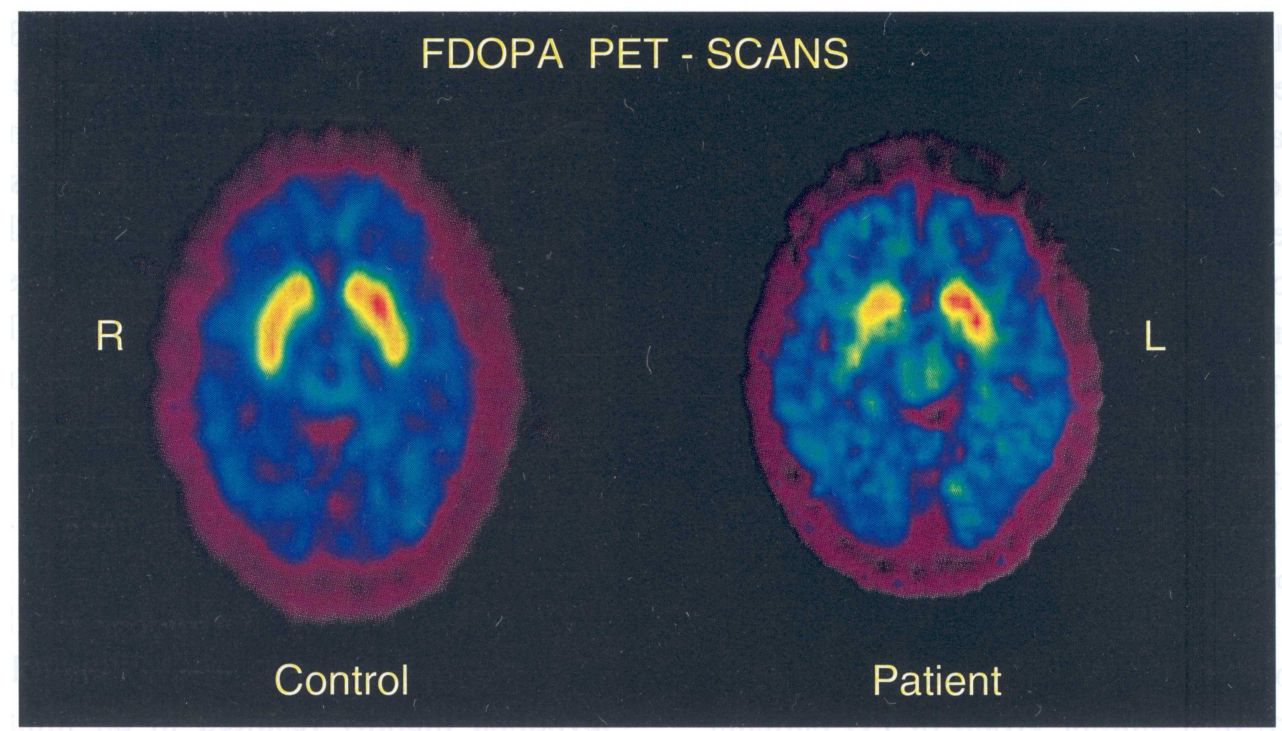

Fig. 1: Transaxial representation of cerebral FDOPA uptake in one healthy volunteer (left panel) and one PD patient (right panel). The front of the brain is on the top of the image. Specific uptake of tracer (see text) is indicated by a linear color scale, red being maximal uptake. The striatum on both sides of the brain is indicated by the high intensity uptake of tracer in the healthy volunteer. It can be seen that in the PD patient the uptake into striatum is diminished, particularly in the more posterior regions.

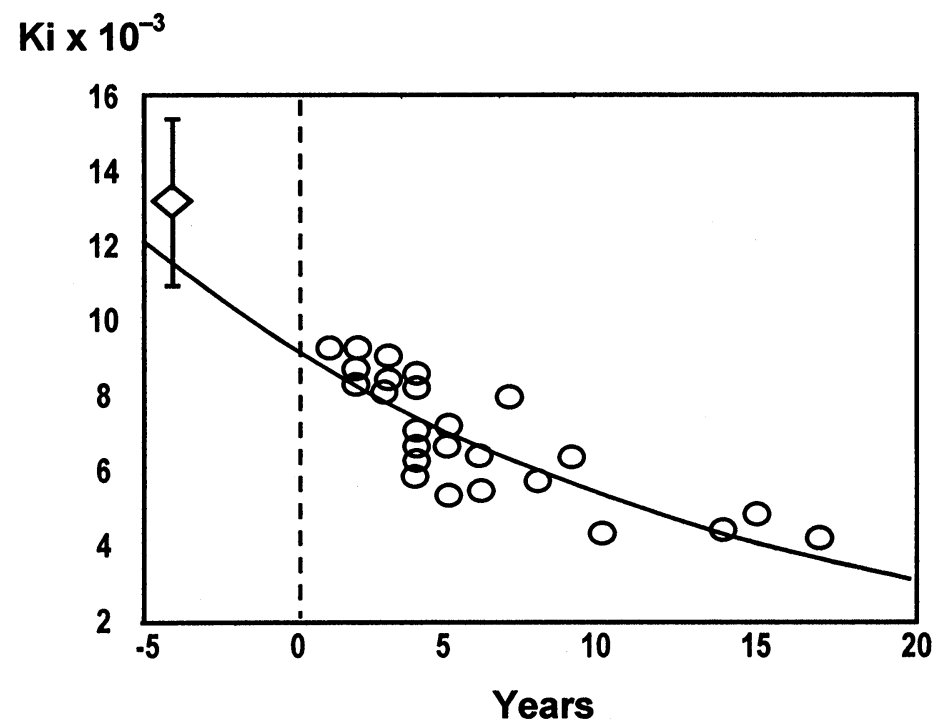

Fig. 2: Correlation between specific FDOPA uptake (expressed as $\mathrm{Ki}$; see text) in putamen and disease duration in patients with PD. Values are compared to healthy age-matched control mean and two SD. Open circles are PD patients and the open diamond is the mean of the controls. 
In the early stages of the disease, when there are only a few signs and symptoms, there are indications that the tracers which measure dopamine reuptake sites (that is, the CIT-derived substances, Benamer et al., 2000) either measured with SPECT or with PET) are more reduced than FDOPA. Later when the disease has been clearly established, the two types of tracer indicate the same degree of striatal dopaminergic impairment. In our own data (to be published separately) it has been shown that [I-123]-FP-CIT had a striatal uptake of $40 \%$ compared with age-matched healthy controls in de novo PD patients with a UPDRS-III motor score of less than 20. On the other hand, FDOPA uptake in a similar group of PD patients was $82 \%$ of healthy control values. One interpretation could be that the dopamine re-uptake sites at the nerve terminals in the striatum of PD patients are downregulated in an early stage of the disease due to the low endogenous dopamine production and, in turn, low dopamine concentration in the synaptic cleft. Since the nerve terminals may still be present at that stage (see above), it is understandable that these different uptake measures are found. It emphasizes that the investigator should know well at which biochemical features he is looking. If this finding is confirmed, then it would mean that in very early stages of PD, the dopamine reuptake tracers might be more sensitive in detecting pathology compared to FDOPA.

\section{GENETIC INFLUENCE ON FDOPA UPTAKE}

Various members of families in which gene alterations have been found have been investigated with FDOPA PET.

\section{Alpha-Synuclein gene}

Eleven subjects of a family were investigated (Kruger et al., 2001). Five were carriers of a mutation of the alpha-Synuclein gene (Ala30Pro point mutation). Of these five subjects, three had PD and two had subtle signs. The symptoms in these patients started at ages 54 to 76. PET scans on two of the symptomatic subjects, two brothers, and on one of the 'non' symptomatic subjects, a sister of the two PD patients, were performed (Kruger et al, 2001). The two brothers (aged 65 and 57), offspring of non-consanguinent parents, presented as patients with dopa-responsive PD. They were found to have the mutant gene 4q21-q23 (alphasynuclein). The oldest brother (65) started with micrographia at age 54. Gradually a more pronounced general hypokinesia developed. Initial levodopa therapy resulted in an improvement of motor signs, but despite additional pharmacotherapy, he developed severe bradykinesia, more pronounced on the left side, rigidity, and moderate postural imbalance. Recently choreatic peak dose dyskinesia, mainly axially, occurred. Up till now tremor, autonomic failure, or a marked cognitive impairment remained absent. The Unified Parkinson's Disease Rating Scale III (Motor Examination Score) was 55 and his Hoehn \& Yahr Stage wàs III-IV (while on medication). The youngest brother (57) suffered from (mainly right sided) hypokinesia and mild limb rigidity since 3 years. Combined pharmacotherapy (levodopa and dopamine agonist) attenuated motor signs successfully, and no other parkinsonian, cognitive, or autonomic signs have occurred till now. The Unified Parkinson's Disease Rating Scale III (Motor Examination Score) was 12 and his Hoehn $\&$ Yahr Stage was II (while on medication). Their sister (52), who also had been tested positive for the mutant gene, had no history of parkinsonism and was clinically unaffected. Their mother, who suffered from parkinsonism, died at the age of 60 . No other family members are known to have movement disorders or related diseases. The two patients had a reduced FDOPA uptake in striatum between $40 \%$ and $54 \%$ of normal control values, 
whereas the sister had normal uptake values. In these subjects, also FDG PET scans which measure regional cerebral glucose consumption, were performed. Several brain regions showed energy consumption deficits in accordance with some neuropsychological deficits. The other brother and even the sister had some FDG uptake impairments. This was also consistent with some deficits apparent at neuropsychological testing.

In summary, the findings in this family indicate that this particular genetic mutation leads to a form of parkinsonism that is very much like the common idiopathic form of PD. In will be of interest to follow the unaffected sister in order to detect in which sequence the clinical and cerebral biochemical impairments will arise.

\section{Parkin gene}

Mutations in the Parkin gene (chromosome 6q25-27) yield an autosomal recessive form of juvenile PD. Exons 3 to 7 are usually affected. A particular characteristic is that no Lewy bodies have been found at post-mortem examination. Two brothers from an Israeli study were investigated with FDOPA PET (Portman et al., 2001). Both brothers were homogenous for large deletions of exon 4,5 , and 6 . They were first degree cousins. The eldest started with clinical signs and symptoms at age 35 and had his PET investigation at age 55 . The youngest brother started at age 28 and was PET investigated at age 49. Their clinical stage was Hoehn \& Yahr Stage IV to V (without medication). FDOPA uptake in putamen was $13 \%$ to $28 \%$ and in caudate nucleus $13 \%$ to $33 \%$ of healthy control values. These findings are similar to those reported earlier (Broussolle et al., 2000). These authors described three non-related patients who had the disease for a very long time and in whom various deletions and mutations of the Parkin gene were found. The FDOPA uptake in putamen in these patients was between $28 \%$ and $44 \%$ of normal.

In summary, it can be said that the abnormalities found with FDOPA PET are different from those found in idiopathic PD: the decreases are more severe and also the caudate nucleus is heavily affected. This would be in concordance with the clinical picture which usually is different from PD, particularly concerning the early age of start. However, a recent paper (Klein et al., 2000) presented a family with Parkin gene mutations in whom the affected family members were clinically indistinguishable from idiopathic sporadic PD.

\section{Twin studies}

One study reported FDOPA results in 18 monozygotic and 16 dizygotic twin pairs, of whom one had PD (Piccini et al., 1999). At baseline the concordance for the FDOPA uptake (reduced in the clinically not-affected twin) was significantly higher in the monozygotic (55\%) than in the dizygotic twin pairs $(18 \%)$. The asymptomatic monozygotic cotwins all showed progressive loss of dopaminergic function over seven years and four developed clinical PD. None of the dizygotic twin pairs became clinically concordant. These findings support some role for genetic factors in the causation of PD. However, the expression of genetic susceptibility must be rather varying since the latencies for clinical concordance vary greatly and may last for many decades. Twin studies have appealing characteristics but also a number of pitfalls, for example, the heavy selection bias and the particular biological situation of the subjects involved. Another recent paper studying a large cohort of twins (Tanner et al., 1999) again found no genetic component to be evident when the disease begins after age 50 years. However, genetic factors appear to be important when the disease begins at or before age 50 years. 


\section{Disease progression}

As PD progresses, specific FDOPA uptake into putamen decreases-this is a marker that the dopamine-producing cells in the substantia nigra projecting to the putamen are continuing to die with relatively the highest rate of decline in the first years of the disease (see Fig. 2).

In the literature, only a few reports are available to assess properly the percentage decline of specific striatal FDOPA uptake per year in an average PD population. The percentages vary between $1 \%$ and $18 \%$, although most figures lie between $5 \%$ and $10 \%$. In healthy controls, the decline is less than $1 \%$ per year. In view of the slow decline of dopaminergic function in PD patients and knowing that in the early phases of the disease a large number of striatal nerve terminals should still be present, albeit malfunctioning, there is therefore a basis to propose neuroprotective strategies if such strategies indeed are available.

\section{Neuroprotection}

Animal experiments have been positive in administering one or other intervention to protect cells from dying or assisting them in recovering from damage. However, to date no such designs have been convincingly successful in man.

Selegiline, a MAO (monoamine oxidase) inhibitor has been extensively tested in, for example, the DATATOP PD study. The basic idea was to block MAO and thus diminish oxidative stress to the dopaminergic system. Selegiline exerts neuroprotection in various cellular and animal models which are independent of MAO-B inhibition but may be related to binding to glyceraldehyde-3phosphate dehydrogenase (Olanow et al., 1998). No apparent benefit in terms of slowing of disease progression in PD patients has been found.

In other studies, dopamine agonists have been proposed to be protective, on the basis of these drugs reducing glutamatergic drive on the substantia nigra. An extensive 5-year study using ropinirol and a 3-year study using pergolide comparing with levodopa use have recently been concluded, and the results will be published shortly. Both studies were performed with clinical scales and longitudinal FDOPA PET scans as outcome measures. In the ropinirol study, 45 patients were included at baseline. After five years, seven patients were still on ropinirole monotherapy and showed a decline of specific FDOPA putamen uptake of in total $12 \%$, whereas the levodopa monotherapy patients $(n=4)$ showed a decline of $15 \%$ in 5 years. The 12 patients with ropinirole and levodopa as rescue therapy showed a decline of in total $36 \%$. The $3-$ year pergolide study included 88 patients. The pergolide monotherapy patients at 3 years showed a putamen FDOPA decline of in total $8 \%$ and the levodopa patients $14 \%$. The difference was not significant.

Thus, no clear-cut beneficial effect in terms of protection against dopaminergic decline has been found for either agonist. In fact, it can be said that levodopa appears to be not deleterious in terms of progression of the disease. Also a dopamine reuptake tracer measured with SPECT is used to study the effects of pramipexol, another dopamine D2 agonist used in everyday treatment of PD patients. The preliminary data appear to be comparable with those of pergolide and ropinirole (personal communication).

Currently a glutamate antagonist, riluzole, is being investigated in de novo PD patients in the context of neuroprotection. Here also, FDOPA PET scans are included in the evaluation of the results. No results are available yet. The advantage of riluzole as compared with deprenyl or dopamine agonists is that the former has no direct clinical symptomatic effect, whereas for the others a combined effect was expected. To disentangle the two effects in multicenter large scale longitudinal studies will be difficult. 
Increased apoptosis has been suggested to be present in the substantia nigra and may be triggered by various mechanisms: oxidative stress, mitochondrial dysfunction, excitotoxicity, excessive concentration of iron (Jenner \& Olanow, 1998). All these unfavorable factors have been demonstrated to be present in the substantia nigra. Selegiline has been proposed (see above) to be a potential candidate to exert neuroprotection since not only does it inhibit MAO-B to reduce oxidative stress but also has it been shown to exert neuroprotection in various cellular and animal models which are independent of MAO-B inhibition but possibly related to binding to glyceraldehyde-3-phosphate dehydrogenase. The activity of the latter enzyme is taken to be an indicator for apoptosis activity. The major problem of disentangling the neuroprotective effect of a substance from its clinical effectiveness is demonstrated also in the case of selegiline. The situation is confounded by the metabolism of selegiline: the desmethylated metabolite is supposed to be protective, whereas the metabolites amphetamine and metamphetamine were shown to be antagonistic to this effect. As a consequence of this TCH346 (formerly CGP 3466B), a propargylamine related to selegiline, has been selected as a more convenient candidate substance. $\mathrm{TCH} 346$ does not lead to MAO-B inhibition, does not metabolite into amphetamines, and is much more potent than selegiline in inhibiting apoptosis. The application of $\mathrm{TCH} 346$ in mice mutant for motoneuron degeneration life span significantly increased, and in an animal model of ischemia, survival of CA1 pyramidal neurons was shown (Andringa \& Cools, 2000). Our own group was involved in testing TCH346 in a group of 8 Rhesus monkeys (results to be published). Sequential bilateral carotid artery application of the dopaminergic neurotoxin MPTP was used as primate Parkinson model. Four hours after the second MPTP lesion, subcutaneous TCH346 administration was started for two weeks twice daily compared to vehicle. The four animals receiving TCH346 did not show any reduction in FDOPA uptake in the corresponding striatum, whereas the vehicle treated animals did show the expected significant decreases. The computerized motor responses of the animals were in complete agreement with the tracer findings.

Apoptosis inhibition is a new target to be tried in humans since in experimental conditions, effectivity against cell damage after toxins like MPTP or after ischemia has been shown. The question remains whether apoptosis is a key factor in the progressive decline of the nigral dopaminergic neurons or whether it is one of the stages in the complex cascades of events once the lesion is taking effect. Most likely it needs to elucidated which factors right in the beginning of the process are responsible for the impaired cell functions before really effective protective or reparative schemes can be tried with some outlook of success.

Restorative therapeutic designs like cerebral implantation of viable possibly modified cells with or without concurrent neurotrophic factors is an option which in the end may certainly bear fruits. Many encouraging experimental findings are available. The recent cell biological work with stem cells adds to the perspectives. However, the level of knowledge and the practical applications in this regard have to date not surpassed the experimental level (Bjorklund \& Svendsen, 2001; Dunnett et al., 2000; Peschanski et al., 1999).

\section{REFERENCES}

Andringa G, Cools AR. 2000. The neuroprotective effects of CGP 3466B in the best in vivo model of Parkinson's disease, the bilaterally MPTP-treated rhesus monkey. J Neural Transm Suppl 60: 215-225.

Benamer HT, Patterson J, Wyper DJ, Hadley DM, Macphee GJ, Grosset DG. 2000. Correlation of Parkinson's disease severity and duration with ${ }^{123}$ I-FP-CIT SPECT striatal uptake. Mov Disord 15: 692-698. 
Bergman H, Feingold A, Nini A, Raz A, Slovin H, Abeles M, Vaadia E. 1998. Physiological aspects of information processing in the basal ganglia of normal and parkinsonian primates. Trends Neurosci 21: 32-38.

Bjorklund A, Svendsen CN. 2000. Chimeric stem cells. Trends Mol Med 7: 1444-1446.

Brooks DJ. 1993. Functional imaging in relation to parkinsonian syndromes. J Neurol Sci 115: 1-17.

Broussolle E, Lucking CB, Ginovart N, Pollak P, Remy P, Durr A. 2000. [ $\left.{ }^{18} \mathrm{~F}\right]$-dopa PET study in patients with juvenile-onset $\mathrm{PD}$ and parkin gene mutations. Neurology 55: 877-879.

Dunnett SB, Nathwani F, Bjorklund A. 2000. The integration and function of striatal grafts. Prog Brain Res 127: 345-380.

Hirsch EC. 2000. Glial cells and Parkinson's disease. J Neurol Suppl II 247: II58-II62.

Hirsch EC, Hunot S, Hartmann A. 2000. Mechanism of cell death in experimental models of Parkinson's disease. Funct Neurol 15: 229-237.

Jenner P, Olanow CW. 1998. Understanding cell death in Parkinson's disease. Ann Neurol Suppl 44: S72-S84.

Klein C, Pramstaller PP, Kis B, Page CC, Kann M, Leung J, et al. 2000. Parkin deletions in a family with adult-onset, tremor-dominant parkinsonism: expanding the phenotype. Ann Neurol 48: 65-71.

Kruger R, Kuhn W, Leenders KL, Sprengelmeyer R, Muller T, Woitalla D, et al. 2001. Familial parkin-sonism with synuclein pathology: Clinical and PET studies of A30P mutation carriers. Neurology 56: 1355-1362.

Leenders KL, Salmon EP, Tyrrell P, Perani D, Brooks DJ, Sager H, et al. 1990. The nigrostriatal dopaminergic system assessed in vivo by positron emission tomography in healthy volunteer subjects and patients with Parkinson's disease. Arch Neurol 47: 1290-1298.

Leenders KL. 1997. Pathophysiology of movement disorders studied using PET. J Neural Transm
Suppl 50: 39-46.

Levy ML, Cummings JL. 1999. Parkinson's disease and Parkinsonism. In: Joseph AB, Young RR, eds, Movement Disorders in Neurology and Neuropsychiatry. Malden: Blackwell Science, Inc.; 171-179.

Melega WP, Grafton ST, Huang SC, Satyamurthy N, Phelps ME, Barrio JR. 1991. L-6- $\left[{ }^{18} \mathrm{~F}\right]$-fluorodopa metabolism in monkeys and humans: Biochemical parameters for the formulation of tracer kinetic models with positron emission tomography. J Cereb Blood Flow Metab 11: 890897.

Olanow CW, Mytilineou C, Tatton W. 1998. Current status of selegiline as a neuroprotective agent in Parkinson's disease. Mov Disord Suppl 13: 5558.

Peschanski M, Defer GL, Dethy S, Hantraye PM, Levivier M, Nguyen JP, Cesaro P. 1999. The need for phase III studies in experimental surgical treatments of Parkinson's disease. Adv Neurol 80: 651-653.

Piccini P, Burn DJ, Ceravolo R, Maraganore D, Brooks DJ. 1999. The role of inheritance in sporadic Parkinson's disease: Evidence from a longitudinal study of dopaminergic function in twins. Ann Neurol 45: 577-582.

Portman AT, Giladi N, Leenders KL, Maguire P, Veenma-Van Der Duin L, Swart J, et al. 2001. The nigrostriatal dopaminergic system in familial early onset parkinsonism with parkin mutations. Neurology 56:1759-1762.

Raz A, Frechter-Mazar V, Feingold A, Abeles M, Vaadia E, Bergman H. 2001. Activity of pallidal and striatal tonically active neurons is correlated in mptp-treated monkeys but not in normal monkeys. J Neurosci 21: RC128.

Tanner CM, Ottman R, Goldman SM, Ellenberg J, Chan P, Mayeux R, et al. Parkinson's disease in twins: An etiologic study. J Am Med Assoc 1999. 281: 341-346. 

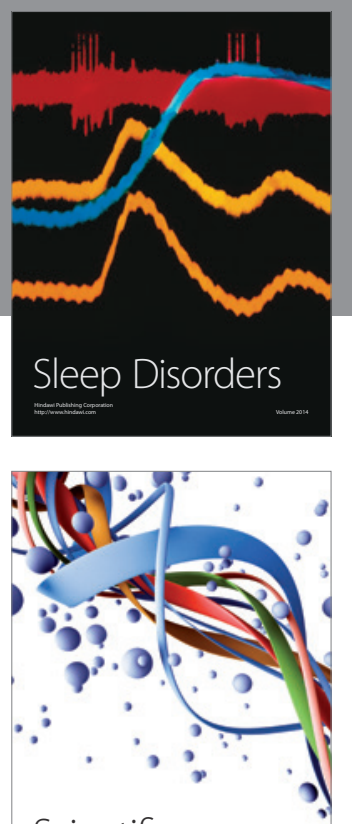

Scientifica
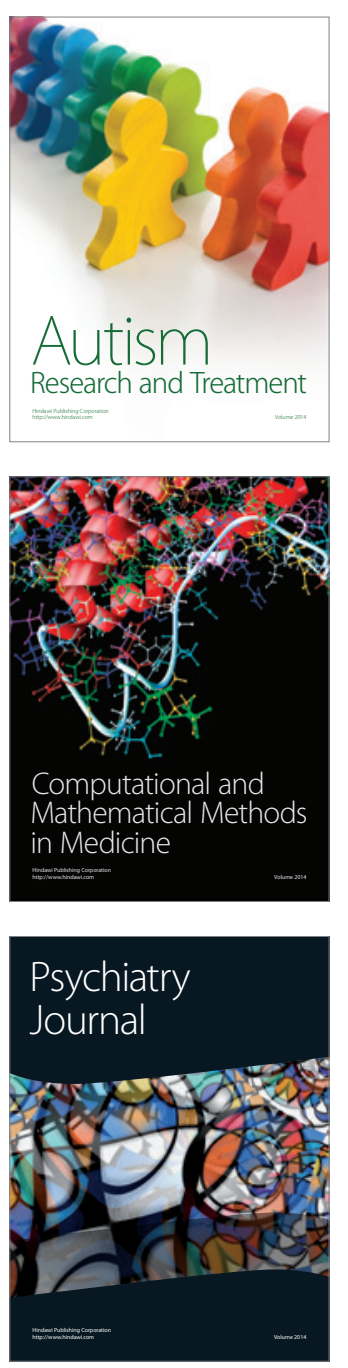
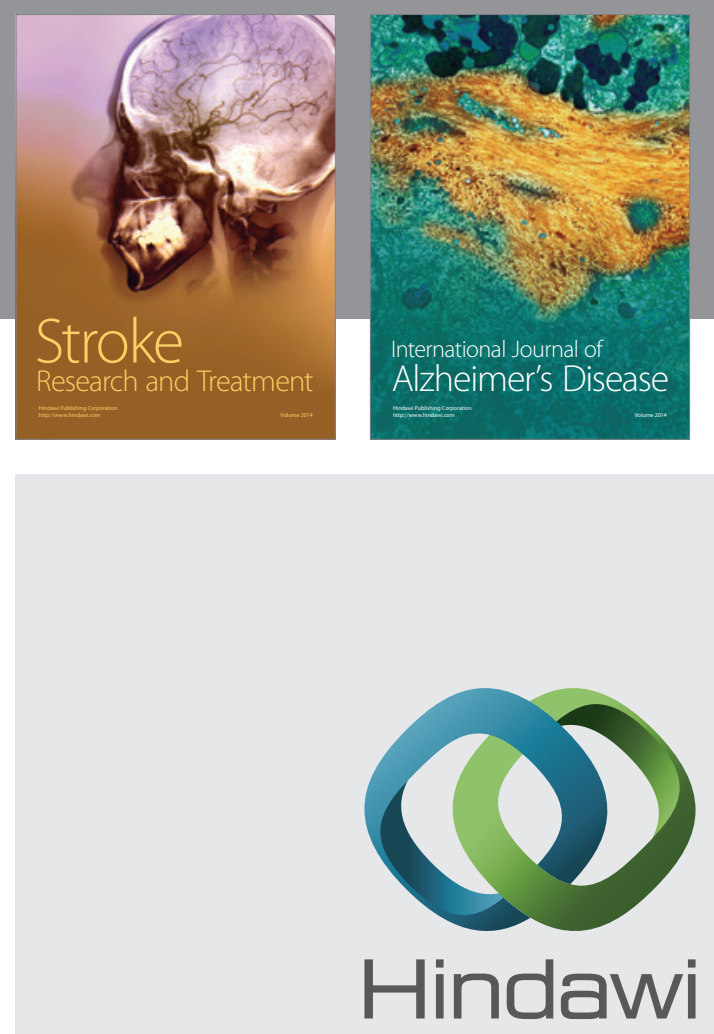

Submit your manuscripts at

http://www.hindawi.com
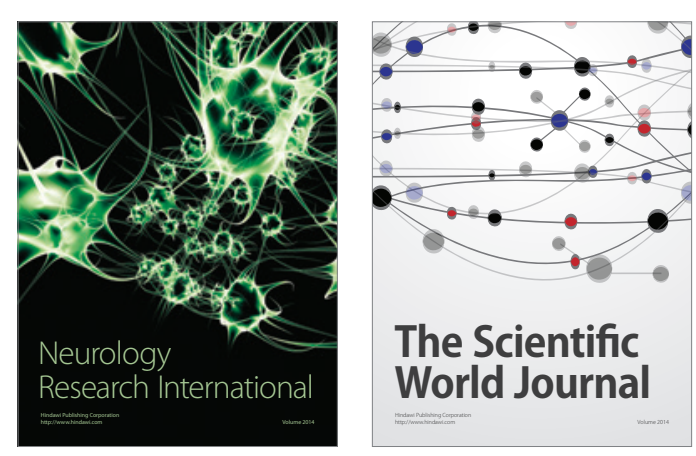

The Scientific World Journal

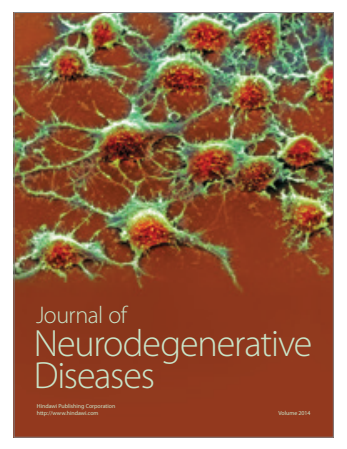

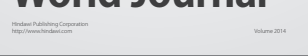

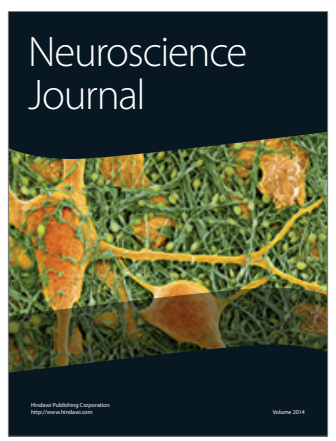

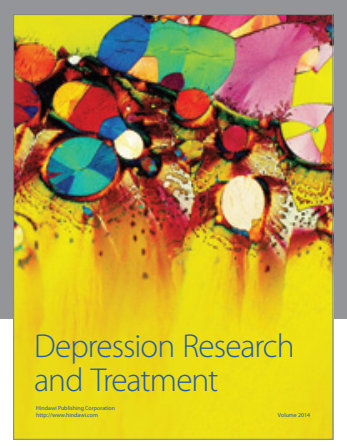
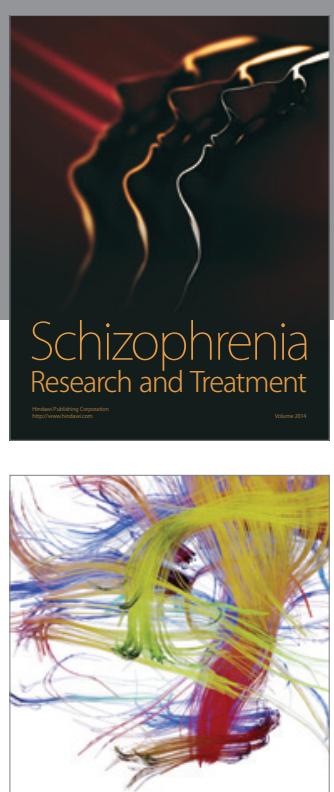

Brain Science

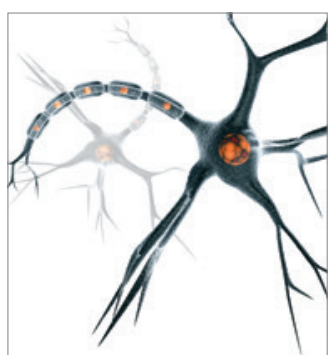

Neural Plasticity
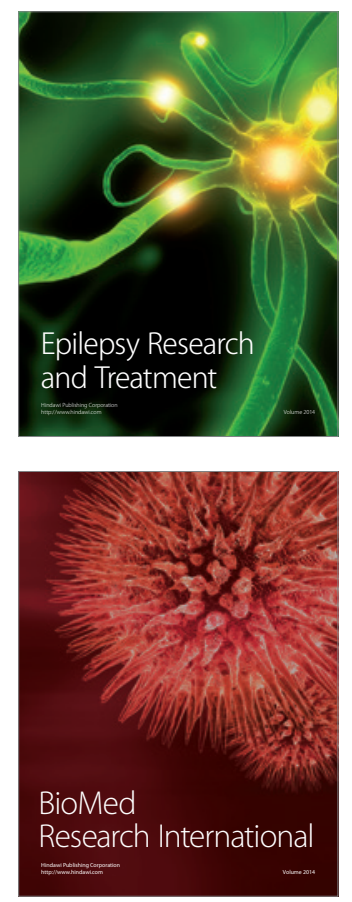

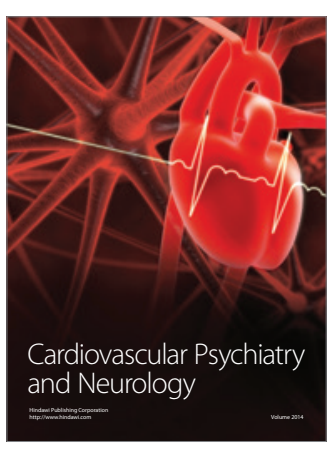

Parkinson's

Disease
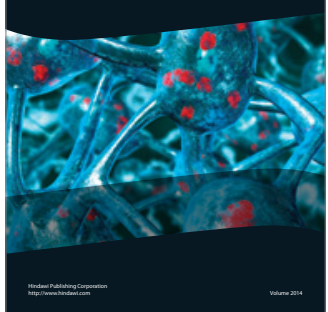